\title{
Análisis de la sentencia: Fernández Ortega vs. México: género, clase y etnicidad
}

\author{
Adriana Rodríguez Caguana*
}

\section{RESUMEN}

El presente trabajo estudiará la sentencia del caso Fernández Ortega contra MéxiCo, dictado en agosto de 2010 por la Corte Interamericana de Derechos Humanos (en adelante Corte IDH) desde las intersecciones de clase, género y etnicidad. El caso Fernández trata sobre la violación sexual que sufrió Inés Fernández, indígena del pueblo Tlapaneco/Me'phaa, por parte de miembros del ejército mexicano en el Estado de Guerrero. Este caso constituye un precedente en la jurisprudencia internacional de derechos humanos. Sin embargo, la Corte IDH no alcanzó a realizar una relación compleja, propia de la interseccionalidad, y se inclinó a entender el caso desde un enfoque de género -considerando solo los instrumentos internacionales de protección de los derechos de las mujeres, como la Convención Interamericana para Prevenir, Sancionar y Erradicar la Violencia contra la Mujer-, dejando relegada la construcción identitaria indígena y sus derechos colectivos. La sentencia nos invita a preguntarnos: ¿es suficiente la teoría de género para entender una violación de los derechos humanos de las mujeres indígenas? PALABRAS ClAVE: género, etnia, lengua, mujeres indígenas, derechos humanos.

\section{ABSTRACT}

This paper shall analyze the judgment from Fernández Ortega vs. México state by the Interamerican Court for Human Rights in August 2010. The study is carried from an integral perspective that includes factors of class, language, gender, and ethnicity that exist in the social anthropology. The Fernandez case involved a sexual rape that the victim had suffered by soldiers. The victim is an indigenous woman from the Tlapaneco/Me'phaa people. This case is very important for the jurisprudence of the Interamerican Court for Human Rights. We presume, however that the Court did not understand the complex relationship between class, language, gender and ethnicity. Finally it favoured only gender perspective, sidelining all other items. Is the Gender Theory sufficient to explain sexual rape and the human rights of indigenous women?

KEYWORDs: gender, ethnicity, language, indigenous women, Human Rights.

* Docente de planta del Área de Derecho de la Universidad Andina Simón Bolívar, Sede Ecuador. 


\section{INTRODUCCIÓN}

L as violaciones de los derechos humanos de las mujeres indígenas necesitan ser estudiadas dentro de la intersección ${ }^{1}$ de clase, género y etnicidad, puesto que la no inclusión de cualquiera de las mencionadas perspectivas imposibilitaría la comprensión integral de la complejidad jurídica. En este sentido, el caso Fernández vs. México sentenciado por la Corte $\mathrm{IDH}^{2}$ se presenta como una oportunidad para entender la violación de los derechos humanos desde una dimensión sociocultural y política. Hay que destacar que en el momento de los hechos la víctima no hablaba español, no había asistido a una educación formal que le permitiera el aprendizaje del idioma oficial, tampoco contaba con control médico -menos aún el ginecológico-, pertenecía a una organización política del pueblo indígena. Si bien la Corte IDH creó un precedente judicial, no pudo superar el análisis parcializado inclinándose por un enfoque de género liberal con cierta sensibilidad para entender la condición identitaria de indígena. Esto es visible porque, además, no se mencionaron en la sentencia ni el Convenio 169, ${ }^{3}$ ni la Declaración sobre Derechos de los Pueblos Indígenas. ${ }^{4}$

El análisis partirá de cuatro categorías que son parte del desarrollo teórico respecto de los derechos humanos de las mujeres indígenas desde el feminismo interseccional. Los puntos que se tomarán como guía para el análisis son los siguientes:

1. La cuestión de género y etnicidad, el contexto de mujeres indígenas sin educación formal y la violación sexual como parte de la historia colonial que han sufrido las mujeres indígenas por parte del "hombre blanco".

2. La cuestión de la educación y la lengua. Una lengua que a lo largo del proceso se verá como desprestigiada y carente de todo valor, incluso burocrático, afectando principalmente a las mujeres indígenas Tlapaneco/Me'phaa.

1. La interseccionalidad es un término feminista desarrollado por la académica estadounidense Kimberlé Williams Crenshaw en sus estudios sobre Teoría Crítica de la Raza. El enfoque de interseccionalidad trata de entender cómo el derecho entiende las múltiples discriminaciones cuando se produce simultáneamente. De esta forma, cuestiona que los casos sean entendidos solo desde una dimensión individual sin entender los otros factores de opresión de las mujeres, como la clase, la etnia y la raza.

2. Corte IDH, Sentencia Fernández vs. México, Serie C, No. 224. Excepción Preliminar, Fondo, Reparaciones y Costas. 30 de agosto de 2010. Interpretación de la Sentencia de Excepción Preliminar, Fondo, Reparaciones y Costas. 15 de mayo de 2011.

3. Convenio 169 de la OIT sobre pueblos indígenas y tribales, 1989. (Entrada en vigor: 5 de septiembre de 1991).

4. Declaración de Naciones Unidas sobre Derechos de los Pueblos Indígenas. (Adoptada en Nueva York el 13 de septiembre de 2007 durante la sesión 61 de la Asamblea General de las Naciones Unidas). 
Al finalizar el artículo se tratará de dar algunos indicios para entender los supuestos teóricos con los que suponemos la Corte Interamericana consideró al momento de entender el caso, desde una perspectiva de género liberal.

\section{LAS MUJERES INDÍGENAS DEL PUEBLO TLAPANECO/ME'PHAA}

El Estado de Guerrero en México es uno de los más pobres del país; posee múltiples dificultades para asegurar a su población derechos sociales básicos, como educación, salud y acceso a la justicia. Dentro de los grupos más afectados por la pobreza se encuentran los pueblos indígenas y, dentro de estos, las mujeres. La mayoría de la población indígena no cuenta con un adecuado servicio de salud y existe un ginecólogo para cada 4.132 mujeres en edad fértil. El 55,7\% de las muertes de mujeres en hospitales ocurren durante el embarazo, el parto o el puerperio. ${ }^{5}$ La población indígena tlapanecos (me' phaa) llega a los 119.291, es decir, el 26\% de la población indígena en Guerrero.

El analfabetismo en los pueblos indígenas en México llega al 50,5\%, siendo las mujeres las más afectadas. El 18\% de la población mayor de 15 años es analfabeta, un $60 \%$ de este total siendo mujeres, ${ }^{6}$ y es el segundo Estado con mayor índice de analfabetismo femenino.

Con estos antecedentes, se evidencia que las violaciones de las mujeres indígenas se produjeron en un contexto de transgresión de los derechos económicos y sociales por parte del Estado de México. Además, el país vive uno de los períodos más violentos de las últimas décadas por el surgimiento de grupos armados provenientes del narcotráfico, un contexto de violencia donde los pueblos indígenas son los más afectados. Inés Fernández denunció la violación sexual ante las autoridades correspondientes; sin embargo, la Procuraduría General de Justicia del Estado de Guerrero declinó su competencia a favor de la Procuraduría General de Justicia Militar, porque los acusados eran parte del ejército mexicano, y la Constitución permitía una jurisdicción militar.

Por otro lado, las pruebas que fueron tomadas por los peritos médicos fueron extraviadas, hecho que entorpeció el proceso de la causa, además Inés no contaba con un intérprete de su lengua materna, lo cual dificultó la denuncia ante la fiscalía y la coherencia de los argumentos.

5. Instituto Nacional de Estadística y Geografía de México 2006.

6. Instituto Nacional de Estadística y Geografía de México 2010. 


\section{LA VIOLACIÓN SEXUAL HACIA LAS MUJERES INDÍGENAS COMO UN ARMA DE GUERRA}

La violación sexual ha sido entendida por la Corte IDH como una experiencia traumática que tiene severas consecuencias; causa gran daño físico y psicológico, deja a la víctima humillada física y emocionalmente. Este delito se asemeja a la tortura por el sufrimiento físico, psíquico y moral agudo. En el presente caso, la Corte IDH volvió a tratar la violación sexual como una práctica de tortura, concluyendo que el Estado violó los derechos de integridad personal, dignidad y vida privada, consagrados en el artículo 5.2, 11.1, 11.2, de la Convención Americana, ${ }^{7}$ en relación al artículo 1 de la misma Convención; y 1,2 y 6 de la Convención Interamericana para Prevenir, Sancionar y Erradicar la Tortura, ${ }^{8}$ así como el deber establecido en el artículo 7 a) de la Convención Interamericana para Prevenir, Sancionar y Erradicar la Violencia contra la Mujer. ${ }^{9}$ Sin embargo, y a pesar de haber ratificado el estándar de la violación sexual como tortura, la Corte IDH se resistió a hacer un análisis más minucioso sobre el alcance sociocultural, histórico y político de la violación sexual como una práctica sistemática colonial y poscolonial contra las mujeres indígenas.

La violación sexual ha sido considerada, en varios estudios en contextos de conflicto armado, como un arma de guerra por parte de las organizaciones de mujeres indígenas. Esta práctica se puede encontrar desde la conquista y colonización hasta la situación actual de las mujeres indígenas en todo el mundo, lo cual nos demuestra que la colonialidad ${ }^{10}$ sigue siendo un tema de actualidad. Un ejemplo de esto es el informe presentado por la Organización Nacional Indígena de Colombia, ONIC, ${ }^{11}$ ante la Representante Especial del Secretario General de las Naciones Unidas para

7. Convención Americana de Derechos Humanos (Pacto de San José). Suscrita en la Conferencia Especializada Interamericana sobre Derechos Humanos. San José, Costa Rica, del 7 al 22 de noviembre de 1969, en vigencia desde el 18 de julio de 1978.

8. Convención Interamericana para Prevenir y Sancionar la Tortura (Adoptada en Cartagena de Indias, Colombia. Fecha: 12/09/85). Entrada en vigor: 02/28/87.

9. Convención Interamericana para Prevenir, Sancionar y Erradicar la Violencia contra la Mujer (Convención de Belén Do Para. (Adoptada en Belém do Pará, Brasil, el 9 de junio de 1994, en el Vigésimo Cuarto período ordinario de sesiones de la Asamblea General).

10. La Colonialidad es una teoría desarrollada por Aníbal Quijano y los estudios latinoamericanos poscoloniales o decoloniales que estudia cómo en el capitalismo latinoamericano persiste la imposición colonial de una clasificación racial/étnica de la población del mundo.

11. Informe: Mujeres indígenas, víctimas invisibles del conflicto armado en Colombia (2011). Informe presentado a la señora Margoth Wallström, Representante Especial del Secretario General para Violencia Sexual en el marco de los conflictos armados. En su visita a Colombia, Bogotá, 16 de mayo de 2012. Disponible en 〈http://cms.onic.org.co/wp-content/uploads/downloads/2012/05/informe-violencia-sexualmujeres-ind\%C3\%ADgenas-Colombia.pdf . 
Violencia Sexual en el Marco de Conflictos Armados. En dicho informe se detalla la crítica situación de tortura sexual de las mujeres indígenas, especialmente de niñas entre 12 y 13 años de edad, en medio del conflicto. La violación sexual se presenta así como un arma de guerra para intimidar a las comunidades. Además, las denuncias no son tomadas en cuenta, no están cuantificadas, no están estudiadas y carecen de importancia social.

El hecho de la indiferencia social e institucional de las violaciones a las mujeres indígenas muestra una naturalización y se constituye como una práctica racista. Tanto las violaciones que se producen en Colombia como en México -o en cualquier país Latinoamericano- parten de la misma problemática donde el racismo colonial hace que la condición del ser indígena y mujer sea más vulnerables a la violencia; además, en ambos casos -Colombia y México- las autoridades tienen como práctica extraviar las muestras periciales, así como la indiferencia en el proceso judicial, lo que lleva a la impunidad de los casos. En Colombia, la ONIC no conoce de sentencias ejecutadas por violaciones sexuales perpetradas hacia mujeres y niñas indígenas. Las escasas denuncias se pueden explicar desde varias dimensiones; por un lado, el miedo a las represalias hace que las mujeres no denuncien los abusos, y, por otro, la indiferencia y la naturalización lleva a las víctimas al silencio y a la desconfianza institucional. Esta característica se repite en otros lugares del mundo en el que existe un contexto de violencia sistemática, siendo la sexualidad de las mujeres indígenas sometida a la tortura. Al mismo tiempo siembra pánico y terror en las comunidades y sus organizaciones políticas. De esta forma, la agresión sexual no solo afecta a las mujeres individuamente, sino que perjudica directamente a toda la comunidad en su dignidad de pueblos.

Por otro lado, es cierto que existe un alcance muy limitado dentro de la Declaración sobre los Derechos de los Pueblos Indígenas en cuanto a los contenidos de los derechos específicos de las mujeres indígenas; únicamente el artículo 22.2 dice: "los Estados adoptarán medidas, junto con los pueblos indígenas, para asegurar que las mujeres y los niños indígenas gocen de protección y garantías plenas contra todas las formas de violencia y discriminación". No obstante esta limitación normativa, existen estudios históricos, antropológicos y documentos recientes que permiten entender la particularidad de los casos en mención. Otro punto que destacar es la violación del derecho a la integridad personal relativo al trato que recibió la señora Fernández en el proceso penal desde la dificultad del idioma hasta la pérdida de las pruebas. La Corte IDH aceptó el allanamiento de México, pero se inhibió de pronunciarse sobre cómo la ausencia de una justicia multilingüe afecta especialmente a las mujeres indígenas que no han sido escolarizadas. Incluso desde una perspectiva positivista, la Corte IDH pudo haberse pronunciado por el artículo 7.1 (derecho a la integridad), 13.2 (Intérpretes en la cuestión administrativa) de la Declaración de las Naciones Unidas sobre los Derechos de los Pueblos Indígenas. 
La ausencia de una justicia intercultural y multilingüe, en zonas donde existe una proporción demográfica considerable de indígenas, demuestra la poca consideración y desvalorización de las lenguas ancestrales, lo que significa también una desvalorización cultural y un impedimento para el acceso a la justicia. Esto corrobora la inexistencia de trabajadores públicos indígenas, lo que responde a una evidente discriminación lingüística en un país que tiene cerca de 68 lenguas originarias. En la misma línea de reflexión podemos observar que la falta de acceso a la educación intercultural bilingüe afecta especialmente a las mujeres indígenas del Estado de Guerrero. Las mujeres suelen ser vistas como las "portadoras de la cultura" o "guardianas de la cultura y de la lengua" sin que exista una real necesidad de su escolarización. El bilingüismo es un derecho de los pueblos indígenas para hacer efectiva la interculturalidad. Sobre este punto la Corte IDH reconoció la violación del acceso a la justicia en condiciones de no discriminación y la falta de protección judicial -artículo 8.1 y 25.1 de la Convención Americana, y el artículo 7.b de la Convención Americana-; sin embargo, no hizo mención de cómo el analfabetismo afectó especialmente a la señora Fernández en su condición de indígena y la falta de sensibilidad del sistema judicial en no tener un intérprete. De esta forma, podemos concluir que la vulneración de las garantías judiciales, especialmente el acceso a la justicia de los pueblos indígenas, está insoslayablemente ligada a los derechos lingüísticos, como el uso de intérpretes $\mathrm{y}$ traductores de lenguas indígenas en los procesos judiciales.

\section{EL DERECHO DE ASOCIACIÓN DE LAS MUJERES INDÍGENAS}

Los representantes de la víctima reclamaron para que la Corte IDH conociera y se pronunciara respecto al derecho a la organización contemplado en el artículo 16 (libertad de asociación en la Convención Americana) porque Inés pertenecía a la Organización Independiente de los Pueblos Mixtecos y Tlapanecos (en adelante OIPMT, organización antecedente de OPIM) desde el año 2000. Las actividades de Inés se encaminaban a mejorar las condiciones de vida de las mujeres de la comunidad, además, su padre había sido un dirigente indígena que fue presuntamente asesinado en situaciones sospechosas. Esta condición de mujer indígena-dirigente era un tema clave en el proceso puesto que la violación sexual pudo haber tenido otros propósitos como la intimidación a las mujeres que hacen dirigencia en la organización.

Es inexplicable la razón por la cual la Comisión Interamericana de Derechos Humanos (instancia que interpone el caso a la Corte IDH) no introdujo en primera instancia el derecho a la libertad de asociación dentro de la demanda. A pesar de esto, la Corte IDH pudo haber conocido el caso, porque la condición de dirigente social en la comunidad la hacía más vulnerable; además, la condición de dirigente estaba directa- 
mente relacionada con la demanda inicial aunque no estuviera señalada expresamente. El pronunciamiento positivo por parte de la Corte IDH hubiera potenciado la relación entre género e indianidad de la víctima; además, hubiera podido pronunciarse en torno al artículo 35 de la Declaración sobre Derechos de los Pueblos Indígenas en cuanto al derecho de organización de los pueblos indígenas.

Por otro lado, la Corte IDH tuvo una hermenéutica formalista cuando también se abstuvo de conocer sobre la muerte del hermano de la víctima, Lorenzo Fernández Ortega, quien fue presuntamente asesinado cuando el caso de Fernández estaba tratándose ante el Sistema Interamericano de Derechos Humanos. La única motivación fue que el caso "no era parte del objeto de la Litis". Desde una perspectiva de interseccionalidad, ambas situaciones son parte de la problemática jurídica, porque la violación ocurrió dentro de un contexto sistemático de violencia hacia los pueblos indígenas.

Una muestra de esta falta de perspectiva integral lo dio el juez ad hoc Alejandro Carlos Espinoza, quien en su voto concurrente fue claro en expresar que la violación sexual no se produjo como un "arma de guerra" -parafraseando al informe de Colombia- ni como una forma de atemorización por parte del Estado a las comunidades indígenas:

6. Debe destacarse que si bien quedó debidamente demostrada la negligencia y falta de resultados a cargo de la procuración de justicia por parte del Estado mexicano, en los diversos fueros constitucionales de carácter competencial en materia penal que se involucraron en la investigación de los hechos, aun como coadyuvantes, también debe indicarse que no se trata de una violación sistémica como instrumento de atemorización dolosa por parte del Estado mexicano respecto de las poblaciones indígenas de la región, particularmente de las mujeres.

7. La demanda como marco litigioso del proceso no excluye la posibilidad para la presentación de pruebas supervinientes previas al dictado de la sentencia, lo que hay que distinguir muy puntualmente de los hechos que no son objeto de litis, no obstante presenten algún tipo de relación con el caso, de modo que la demanda o escrito inicial fija la Litis.

\section{LOS LÍMITES DEL ENFOQUE DE GÉNERO EN LA SENTENCIA FERNÁNDEZ}

El concepto "género" es uno de los grandes aportes del feminismo contemporáneo occidental. Sin embargo, el término ha sido tomado por el feminismo para universalizar los valores construidos por la cultura occidental, alejados de otros feminismos que se construyen en contextos de diversidad lingüística, cultural y religiosa, conocidos como "Feminismo de la diferencia". En otras palabras, cuando hablamos de feminismo o "género" solemos pensar en un movimiento histórico que permitió consolidar el 
derecho al voto de las mujeres, y de un movimiento actual, que ha puesto en debate el derecho a decidir sobre nuestros cuerpos, especialmente nuestros úteros sometidos a la decisión del Estado heteropatriarcal capitalista. Pareciera como si el feminismo contemporáneo se agotara en estas raíces liberales, que son legítimas, pero no universales. Las mujeres no-occidentales reclaman la construcción de otro tipo de derechos. En la perspectiva del sur global ha emergido un feminismo anticolonial y antirracista de las mujeres afrodescendientes, quienes han tomado la dirección.

Para Stolcke ${ }^{12}$ la categoría género no puede estar separado de otras, como el de clase o etnia, puesto que existen diferencias sociales, culturales y económicas que deben ser tomadas cuentan en sociedades diversas; la diferencia social y cultural determinará mayor o menor grado de vulnerabilidad; en otras palabras, una mujer blanca, occidental y privilegiada económicamente es menos vulnerable socialmente que la mujer india-negra y pobre. El problema de concebir al "género" desde una perspectiva liberal, aislado de la condición de clase y origen cultural, tiene como efecto una interpretación sesgada por las luchas civiles y políticas de las mujeres occidentales sin que se produzca una elaboración teórica más profunda sobre los derechos de género desde la indianidad.

Estos problemas se hacen visibles cuando hay casos de violaciones de derechos humanos de las mujeres indígenas, y se complica más cuando los perpetradores de los abusos son agentes del Estado. En la práctica se ha demostrado que los discursos se superponen y el juez tiende a optar por los derechos individuales en lugar de los derechos colectivos. En el caso Fernández, la Corte IDH se inclinó por una interpretación de vulneración de los derechos individuales con un cierto interés por entender las dimensiones de la indianidad, pero nada más. Los instrumentos utilizados para sancionar el caso fueron tomados de las reivindicaciones de los derechos a la luz de concepto género liberal.

La falta de esta perspectiva, tiene un origen también teórico de los fundamentos de los derechos, puesto que cuando se superponen discursos como el de los derechos individuales (como el género) y los colectivos (identidades indígenas), las cortes apuestan por aquellos que han sido considerados como los "auténticos" derechos, los individuales. En definitiva, esto muestra la tensión permanente entre el discurso liberal, tradicionalmente individualista frente al discurso desarrollado por los derechos colectivos de los pueblos indígenas. La sentencia Fernández vs. México confirma que

12. Verena Stolcke, “¿Es el sexo para el género lo que la raza para la etnicidad... y la naturaleza para la sociedad?", Política y Cultura, No. 14, Universidad Autónoma Metropolitana/Xochimilco Distrito Federal (2000). 
se puede sancionar un caso de violación a los derechos de la mujer desde una perspectiva liberal.

Esta limitación hizo que la Corte IDH no pudiera pronunciarse por otros sucesos que evidentemente eran parte de la complejidad del caso, como el presunto asesinato del hermano y del padre de la víctima, así como los derechos políticos comunitarios de la mujer-dirigente indígena. Fijar la litis desde una motivación formalista obstaculizó el análisis complejo del caso y limitó la posibilidad de construir estándares más firmes para los derechos de las mujeres indígenas. La Corte IDH sentenció al Estado mexicano a que sancione a los culpables, otorgue becas de estudio a las mujeres y contrate traductores en las instituciones públicas. Estas reparaciones no cuestionan la discriminación y el racismo, tanto de los perpetradores de las violaciones como de las instituciones públicas indiferentes.

Es pertinente lo señalado por Sieder y Sierra ${ }^{13}$ a través de la investigación Acceso a la justicia para las mujeres indigenas en América Latina, donde concluyen que no puede concretarse una perspectiva de género sin los derechos colectivos, puesto que la aplicación por sí sola de dicha perspectiva es limitada y no alcanza a cubrir las necesidades reales de las mujeres indígenas. En este sentido, las autoras concuerdan que solo en la medida en que se respeten los derechos colectivos de los pueblos indígenas se podrá garantizar el cumplimiento de los derechos de las mujeres indígenas a la justicia y a su identidad cultural.

El analfabetismo de Inés, la falta de servicios de salud, la violación sexual, la indiferencia institucional por la denuncia, la pérdida de las pruebas médicas, la falta de traductor, la condición de dirigente indígena y el asesinato del padre y del hermano son hechos que no corresponden a un solo caso, sino que se repiten en los contextos de violencia sistémica donde los cuerpos de las mujeres indígenas son torturados por su condición étnica. La violación sexual como arma de guerra tampoco es nueva. No existe un solo período desde la conquista-colonización hasta en la actualidad en el que las mujeres indígenas hayan vivido sin el constante miedo a ser víctimas de violencia. Si revisamos la historia en la región, especialmente de las mujeres dirigentes, no es difícil encontrarse con relatos que detallan los abusos perpetrados por los hombres blancos y mestizos, hechos que fueron naturalizados por toda la sociedad que legitimaba las relaciones de explotación clasistas y raciales. Termino este análisis con una frase de Sieder y Sierra que invita al debate entre quienes nos consideramos feminis-

13. Rachel Sieder y María Teresa Sierra, "Acceso a la justicia para las mujeres indígenas de América Latina". Este artículo se preparó como un documento de trabajo para el informe de UNIFEM Progress of the World's Women-Access to Justice. Financiado por el Norwegian Research Council (Proyecto No. 199745/ S50), 2011. 
tas: "Las mujeres indígenas no están sujetas a la violencia física y sexual debido a su género, sino debido a su etnicidad, su clase social y a su historia”.

\section{BIBLIOGRAFÍA}

ONIC, Organización Nacional Indígena de Colombia. "Mujeres indígenas, víctimas invisibles del conflicto armado en Colombia". Informe presentado a la señora Margoth Wallström Representante Especial del Secretario General para Violencia Sexual en el marco de los conflictos armados. En su visita a Colombia, Bogotá, 16 de mayo de 2012. Disponible en 〈http://cms.onic.org.co/wp-content/uploads/downloads/2012/05/informe-violencia-sexualmujeres-ind $\% \mathrm{C} 3 \%$ ADgenas-Colombia.pdf .

Sieder, Rachel, y María Teresa Sierra. “Acceso a la justicia para las mujeres indígenas de América Latina”. Este artículo se preparó como un documento de trabajo para el informe de UNIFEM Progress of the World's Women-Access to Justice. Financiado por el Norwegian Research Council (Proyecto No. 199745/S50), 2011.

Sipaz, Servicio Internacional para la Paz. 〈www.sipaz.org〉, visitado por última vez el 15 de julio de 2012.

Stolcke, Verena. “¿Es el sexo para el género lo que la raza para la etnicidad... y la naturaleza para la sociedad?". Política y cultura, No. 14. Universidad Autónoma Metropolitana/Xochimilco Distrito Federal (2000).

\section{Otros}

CIDH, Corte Interamericana de Derechos Humanos. Sentencia Fernández Ortega y otros vs. México. Sentencia del 30 de agosto de 2010.

Convención Interamericana para prevenir, sancionar y erradicar la tortura. Adoptada en Cartagena de Indias, Colombia, el 9 de diciembre de 1985, y entrada en vigor el 28 de febrero de 1987.

Convención Interamericana para prevenir, sancionar y erradicar la violencia contra la mujer. Adoptada y abierta a la firma en Brasil el 9 de junio de 1994, y entrada en vigor el 5 de marzo de 1995.

Declaración sobre los Derechos de los Pueblos Indígenas. Adoptada el 13 de diciembre de 2007. 